University of Rhode Island

DigitalCommons@URI

Open Access Master's Theses

1999

\title{
The Effect of Vitamin E Supplement on Liver Mitochondrial \\ Profiles in Mice
}

Karen Ann Agostinucci

University of Rhode Island

Follow this and additional works at: https://digitalcommons.uri.edu/theses

\section{Recommended Citation}

Agostinucci, Karen Ann, "The Effect of Vitamin E Supplement on Liver Mitochondrial Profiles in Mice" (1999). Open Access Master's Theses. Paper 931.

https://digitalcommons.uri.edu/theses/931

This Thesis is brought to you for free and open access by DigitalCommons@URI. It has been accepted for inclusion in Open Access Master's Theses by an authorized administrator of DigitalCommons@URI. For more information, please contact digitalcommons-group@uri.edu. 
THE EFFECT OF VITAMIN E SUPPLEMENTATION ON LIVER MITOCHONDRIAL PROFILES IN MICE BY KAREN ANN AGOSTINUCCI

A THESIS SUBMITTED IN PARTIAL FULFILLMENT OF THE REQUIREMENTS FOR THE DEGREE OF MASTER OF SCIENCE

IN

EXERCISE SCIENCE 
MASTER OF SCIENCE THESIS

OF

KAREN ANN AGOSTINUCCI

APPROVED:

Thesis Committee:

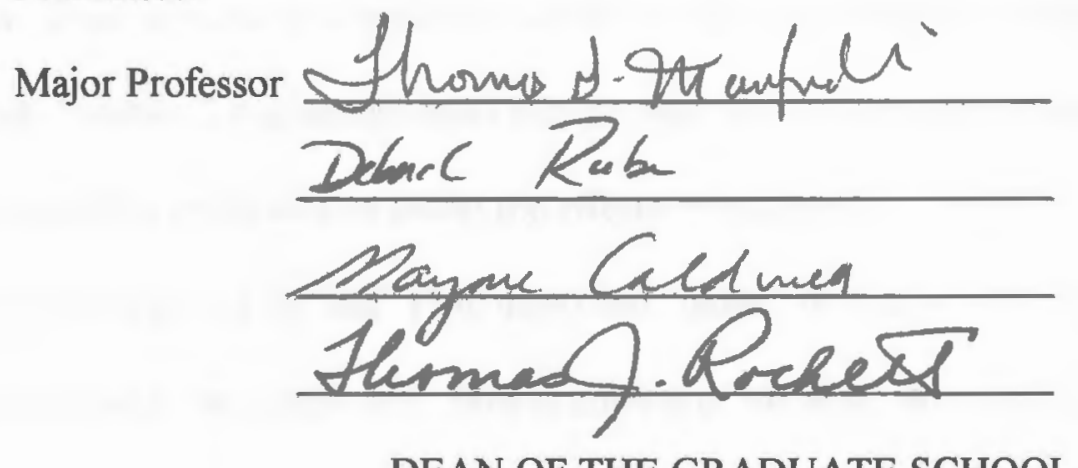

DEAN OF THE GRADUATE SCHOOL

UNIVERSITY OF RHODE ISLAND

1999 


\section{ABSTRACT}

Cell oxidative damage caused by free radical production during respiration plays a major role in tissue dysfunction. Mitochondria are subcellular sites for oxidative phosphorylation and are the major source of free radicals. Their high oxidative activity makes them susceptible to oxidative damage. Antioxidants protect the cell against oxidative damage, but when defenses are not adequate cells are subjected to oxidative stress. A lack of vitamin E, and selenium results in enlargement of liver mitochondria, suggesting mitochondrial fusion, and an inability to divide. Recent studies suggest that aging is associated with mitochondrial enlargement and elongation, and that these mitochondria are less oxidatively efficient, and less resistant to oxidative damage. Vitamin E supplementation may protect against these age-related effects. The purpose of this study was to assess the effects of vitamin $\mathrm{E}$ supplementation (500 IU/kg diet) on "old" (760 days) and "older" (827 days) C57BL/6 mice liver mitochondrial (Mt) morphometry. Measurements of Mt area, short and long axis, and size distributions were taken from control and vitamin E supplemented mice. Transmission electron microscopy and image analysis were used to measure fixed mitochondria from liver homogenized samples. We hypothesized that vitamin E supplementation will result in smaller, more round liver mitochondria. The "old" vitamin E supplemented mice had significantly smaller $(p<.0001)$ liver mitochondria than age-matched controls. Age had no significant effect on Mt area and short axis in the vitamin $E$ supplemented mice. Age had a significant effect $(p<.002)$ on the long 
axis in the vitamin $\mathrm{E}$ supplemented mice. Mt size distributions ( $\mathrm{N}=10$ sizes) of area showed that the five smallest sizes constituted $73.9 \%, 78.1 \%$, and $53.2 \%$ of the total Mt population in the "old" vitamin E (E), "older" vitamin E (OE), and "old" control (C) mice, respectively. Size distributions of the long axis showed that the smallest five sizes constituted $61.2 \%, 45 \%$, and $30.8 \%$ in the $\mathrm{E}, \mathrm{OE}$, and $\mathrm{C}$ mice, respectively. Regarding distributions of the short axis, the smallest five sizes constituted $51.1 \%$, $45 \%$, and $17 \%$ in the $\mathrm{E}, \mathrm{OE}$, and $\mathrm{C}$ mice, respectively. Analysis of the ratio of the long axis to short axis showed that age had a significant effect on mitochondrial elongation in the vitamin $E$ fed mice. These data suggest that 1) vitamin $E$ supplementation results in smaller liver mitochondria in mice when compared to age-matched cohorts, and 2) aging elongates but does not alter the size of liver mitochondria in mice supplemented with vitamin $\mathrm{E}$. These results suggest that vitamin $\mathrm{E}$ may provide a protective effect against age-related mitochondrial fusion and enlargement. 


\section{ACKNOWLEDGEMENTS}

There have been many people responsible for the successful completion of this thesis. I would like to especially thank my major professor, Dr.Thomas Manfredi, for his invaluable advice and guidance through this process. The many hours of attention he gave this project are greatly appreciated. I would also like to thank my committee members, Dr. Marjorie Caldwell and Dr. Debra Riebe.

This would not have been possible without the support of my family. My mom, dad, and grandparents have always stressed the importance of education and have supported all my educational endeavors. My three children, Julie, Kevin, and Kristen, have been so patient as mom has dedicated much time to her own school work. And lastly, my husband Jim, whose unending love, support and encouragement carried me through the difficult times when I needed it most. His knowledge in research has contributed greatly to this paper. 


\section{PREFACE}

The purpose of this study was to examine the effect of vitamin $E$ supplementation on liver mitochondrial profiles in mice. This thesis is written in manuscript format for submission to the AGE journal for publication. 


\section{TABLE OF CONTENTS}

\section{PAGE}

ABSTRACT

ACKNOWLEDGMENTS

PREFACE

v

TABLE OF CONTENTS

vi

LIST OF TABLES

viii

LIST OF FIGURES

ix

\section{MANUSCRIPT}

INTRODUCTION

1

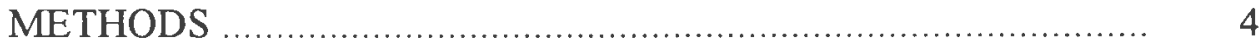

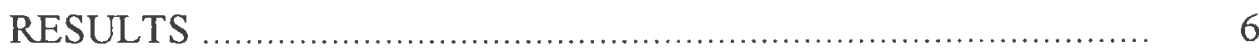

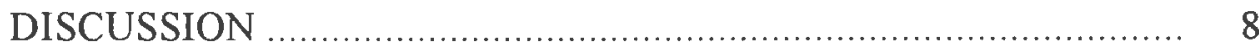

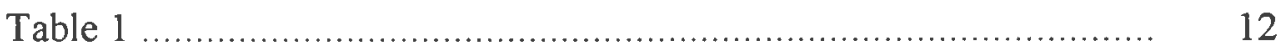

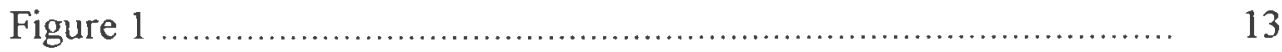

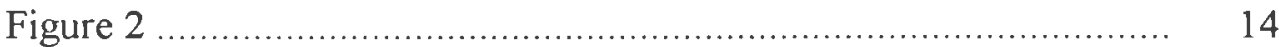

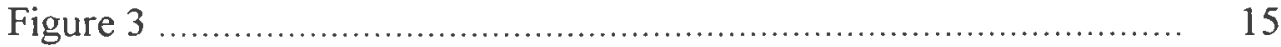

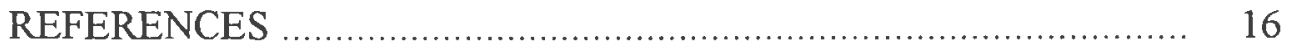

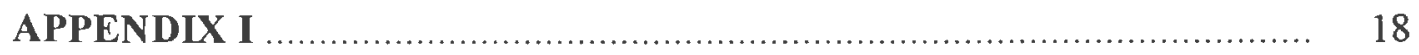

\section{LITERATURE REVIEW}

Mitochondria and Aging 
PAGE

Vitamin E Supplementation ...................................................... 21

Vitamin E and Exercise ............................................................. 23

Vitamin E Deficiency ................................................................ 24

Mitochondrial Intervention Studies ................................................ 26

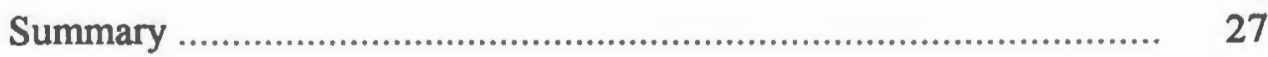

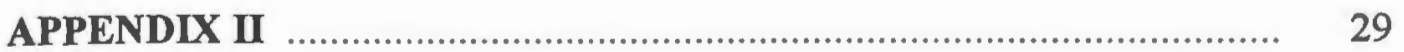

Statement of the Problem ......................................................... $\quad 30$

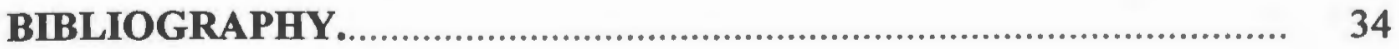




\section{LIST OF TABLES}

PAGE

TABLE 1. Vitamin E and mitochondrial morphometry

12 


\section{LIST OF FIGURES}

PAGE

FIGURE 1. Mitochondrial area-pooled cumulative data ............................ 13

FIGURE 2. Mitochondrial sizes: long axis-pooled data ............................. 14

FIGURE 3. Mitochondrial sizes: short axis-pooled data ............................... 15 


\section{INTRODUCTION}

Aging is a consequence of cellular damage to DNA, proteins and lipids caused by accumulative effects of reactive oxygen species. Mitochondria are the sites of oxidative phosphorylation and are major targeted sites for oxidative damage. Despite the high rate of tissue mitochondrial turnover, mitochondrial function declines with age as a result of damage caused by endogenously produced oxidants (Ames, Shigenaga, and Hagen, 1993; Shigenaga, Hagen, and Ames, 1994; Zorov, 1996).

Mitochondria undergo morphological changes as aging progresses from maturation to senescence. Mitochondrial division during maturation increases the surface-to-volume ratio, indicated by a shift toward a greater percentage of smaller mitochondria. Following maturation, mitochondria show evidence of lipid membrane damage, leading to cytochrome $\mathrm{C}$ leakage into the cytosol, triggering events leading to apoptosis (Ji and Leichtweis, 1997). Morphological indicators of apoptosis include a significant decrease in the total number of mitochondria associated with enlargement and elongation of these organelles ( $\mathrm{Ji}$ and Leichtweis, 1997). Bertoni-Freddari and associates (1993) examined cerebellar glomeruli of young, adult, and old rats and found that the number of mitochondria decreases with age as size and elongation of these organelles increases. Cosmas et al.(1994) found that with senescence there is a shift in size distribution to a greater percentage of enlarged mitochondria in rat myocardium. These findings suggest that with age mitochondria increase in size and elongate.

Interventions including diet and exercise can alter mitochondrial morphology.

Forty percent caloric restriction has been reported to decrease skeletal muscle 
mitochondrial mean area in older mice. (Utley et al., 1999). The caloric restricted mice showed evidence of smaller, more rounded mitochondria when compared to their agematched controls, supporting the hypothesis of maintenance of mitochondrial plasticity with age in diet restricted mice. These findings also suggest that the use of stereological methods is a less sensitive indicator of mitochondrial plasticity when compared to image analysis. This conclusion was based on findings that the volume density of mitochondria increased with age in ad libitum and diet restricted mice suggesting an increase in mitochondrial mass when estimated with stereological techniques.

Enhanced mitochondrial fusion during senescence suggests that mitochondrial plasticity may be compromised at old age. Cosmas and Manfredi (1995) reported that exercise training during maturation and middle age dramatically increased the proportion of smaller size mitochondria in rat myocardium. They concluded that cellular mechanisms respond to physical training by alterations in mitochondrial morphology which would be more efficient to handle the increased metabolic demand. This would help prevent the increased oxidative stress associated with physical training. However, beyond 450 days a "threshold age" can be defined in which initiation of rigorous exercise training fails to increase the population of small mitochondria (Cosmas et al., 1996). This finding is further substantiated by Kiesling et al. (1973) who quantified mitochondria morphology in muscle from young and old trained humans after physical training. The authors found that both young and old subjects increased mitochondrial volume following training, but the young trained responded by 
an increase in number of mitochondria and the older subjects responded with an increase in mitochondrial size.

Cellular defense mechanisms protect against oxidative damage by preventing the formation of reactive oxygen, or by destroying already formed oxidants (Zorov, 1996). These defenses include endogenous enzymatic and dietary antioxidants (Ames et al., 1993). Vitamin $\mathrm{E}$ is an effective antioxidant because it protects against oxidative stress and contributes to cellular membrane stability. Epidemiological evidence suggests that vitamin E might decrease the prevalence of cardiovascular disease and certain cancers (Meydani, 1995). Meydani et al. (1993) suggested that vitamin E protects against oxidative damage which can occur during exercise. Deficiencies in vitamin $\mathrm{E}$ have been shown to have a harmful effect on the immune response in aged individuals. Supplementation to levels above the recommended daily allowance has enhanced immune function in several studies (Meydani et al., 1995; Meydani \& Hayek, 1995). Frigg \& Rohr (1976) examined the effect of vitamin $E$ on liver mitochondria of male mice and found that vitamin E deficiency increases the surface density of the mitochondrial inner membrane, however cytochrome density was reduced. This study suggested that vitamin E deficiency was associated with increased formation of defective mitochondrial membranes. Since vitamin $\mathrm{E}$ has been shown to be an effective antioxidant, the intent of this study was to examine the effects of vitamin $E$ supplementation on liver mitochondrial size, elongation, and size distributions. 


\section{METHODS}

Eight experimental and four control C57BL/6 mice were randomly drawn from a large scale nutritional intervention study (Lipman et al., 1998). One hundred sixtyeight mice were bred and raised under barrier conditions and housed in autoclaved, filtered cages under a 12/12/ light dark cycle. At age 18 months (540 days), the mice were fed ad libitum modified AIN 76 semisynthetic diet or modified AIN 76 diet supplemented with one of the following antioxidants: vitamin E (470 ppm), glutathione, vitamin E plus glutathione, melatonin, or strawberry extract. The control diet contained $30 \mathrm{ppm}$ of vitamin $\mathrm{E}$. The supplemented mice received an additional 470 ppm of vitamin E (DL- $\alpha$-tocopheryl acetate) in their diet increasing the intake per gram of body weight by $0.07 \mathrm{mg}$. Prior to 18 months of age, mice were fed autoclaved chow PMI 5021. The control group was sacrificed at 760 days of age. The experimental group was sacrificed at 760 days ("old") and 827 days ("older") of age. Four "old" control, four "old" experimental, and four "older" experimental animals were used in this study.

The ultracentrifuged mitochondrial pellets were fixed in modified Karnovsky's fixative, rinsed in buffer, dehydrated in alcohol and infiltrated with LR White embedding media. Ultra-thin sections were cut using a Dupont Sorval MT2-B ultramicrotome and stained with uranyl acetate. Five grids were taken from each animal. Each grid was separated by a thick section (approximately 10 microns). This protocol assured random sampling and that the samples were representative of that animal (Baudhuin \& Berthet, 1967). 
Grids were observed with a Philips 300 transmission electron microscope (TEM) at a magnification of $3400 X$. The TEM was equipped with an Advanced Microscopy Techniques imaging capturing system consisting of a retractable mirror and a Dage 72 CCD camera. Images of mitochondria were captured at $1200 \mathrm{X}$ with a Data Translation image grabbing board housed in a Macintosh IIci computer, using NIH 5.56 image analysis software. Images were stored as TIFF files on an Iomega Zip drive and analyzed on a Dell Pentium II 350 PC using NIH Image 1.60 software.

Mitochondria were identified by a protocol used in a previous study (Baudhuin \& Berthet, 1967). The inner membrane of the mitochondria had to be recognized and characteristic internal cristae had to be present. When identification by this protocol was not achieved, the profile was not measured. Mitochondrial area, short axis, and long axis were measured in micrometers using the NIH Image analysis software. Approximately $156 \pm 90$ mitochondria were measured per animal, with four animals per group. An Analysis of Variance model for pooled data was used to test for significant differences due to diet and age. Area, short axis, and long axis size distributions were derived for the "old" control, "old" vitamin E, and "older" vitamin E mice using ten sizes. Measurement reliability was demonstrated by taking a random image with 82 identified mitochondria and repeating the measurements. Intraclass Correlation Coefficient $(3,1)$ was used to calculate measurement reliability for area, long axis, and short axis.

Standard microscopic techniques for validity were performed. Resolution was checked by asymmetry of the microscopic column using a holy grid. Magnification was 
validated by a carbon grated calibrated grid. Calibration of the TEM objective lens was done by using a grating replica photographed under the same conditions as the specimen grid.

\section{RESULTS}

\section{RELIABILITY OF MEASUREMENTS}

The Intraclass Correlation Coefficients $(3,1)$ for area, long axis, and short axis were $0.92,0.89$, and 0.91 respectively.

\section{MITOCHONDRIAL MEAN DATA}

Diet had a significant effect on mitochondrial area when comparing the "old" 760 day control and vitamin $\mathrm{E}$ supplemented animals (Table 1). The vitamin $\mathrm{E}$ supplemented mice had a smaller mean mitochondrial area than age-matched controls $(p<.0001)$. Diet also had a significant effect on the long axes $(p<.0001)$, and the short axes $(p<.0001)$. The "old" vitamin E supplemented animals had significantly smaller long, and short axes when compared to their age-matched controls. Age had no effect on the mean mitochondrial area of the vitamin E supplemented mice. Age had no effect on the mean short axis of the vitamin E supplemented mice, however the mean long axis did increase significantly $(p<.002)$.

\section{MITOCHONDRIAL SIZE DISTRIBUTION: AREA}

Mitochondrial area measurements (Figure 1) were grouped into ten sizes, which 
were further grouped into three categories consisting of "small" (sizes 1-3), "medium" (sizes 4-6), and "large" (sizes 7-10) mitochondria. The greatest differences between diet and age groups appeared in the "small" size category. The "old" vitamin E and "older" vitamin E supplemented mice had $52 \%$ and $38 \%$ of their total mitochondrial populations fall into this category, respectively. The "old" control mice had $18 \%$ of the total mitochondrial population fall into the "small" category. In the "medium" size mitochondria, the "old" and "older" vitamin E supplemented mice, and the "old" controls had $28 \%, 50 \%$, and $48 \%$ of the total mitochondrial populations fall into this category, respectively. In the "large" size mitochondria, the "old" and "older" vitamin E supplemented animals, and the "old" controls had $15 \%, 11 \%$, and $22 \%$ of their total population fall into this category, respectively.

\section{MITOCHONDRIAL SIZE DISTRIBUTION: LONG AND SHORT AXIS}

The size distributions for the mitochondrial long axis (Figure 2) show that the "old" vitamin E supplemented animals had 13\%, $62 \%$, and $24 \%$ of their total mitochondrial population fall into the "small", "medium", and "large" categories, respectively. The "older" vitamin E supplemented animals had $2 \%, 72 \%$, and $24 \%$ of their total mitochondrial population fall into the "small", "medium", and "large" categories, respectively. The "old" controls had 5\%, 50\%, and $38 \%$ if their total mitochondrial population fall into the "small", "medium", and "large" categories, respectively. Similar age and dietary trends in mitochondrial size categories were evident when measurements were taken of the short axis (Figure 3). The "old" control 
mice had $52 \%$ of their mitochondria fall into the "large" category compared to about $27 \%$ in the "old" and $26 \%$ in the "older" vitamin E supplemented mice. The "old" vitamin E supplemented mice had the greatest number of mitochondria in the "small" category compared to the other subject groups. In the "medium" category, the "older" vitamin $\mathrm{E}$ supplemented animals had $71 \%$ of their mitochondria fall into this category compared to $58 \%$ in the "old" vitamin E animals, and $44 \%$ in the "old" controls.

\section{RATIO: LONG AXIS TO SHORT AXIS}

To determine if age, and vitamin $\mathrm{E}$ had an effect on mitochondrial shape, the ratio of the long axis to the short axis was analyzed. Individual ratios were calculated for each mitochondria evaluated in this study. The mean long axis to short axis ratios for "old" vitamin E, "older" vitamin E and "old" controls were 1.17, 1.26, and 1.17, respectively. A t-test was used to determine significance. Vitamin E did not have a significant effect on elongation when comparing "old" vitamin E supplemented mice to age-matched controls. Age had a significant effect $(p<.001)$ on mitochondrial elongation with the vitamin $\mathrm{E}$ supplemented mice.

\section{DISCUSSION}

Bertoni-Freddari and associates (1993) examined cerebellar glomeruli of young, adult, and old rats. They investigated mitochondrial volume density, numerical density, average volume, and average length. The investigators found that volume density remained constant throughout life, however numerical density decreased from adult to 
old rats. Measurements of mitochondrial area and diameter supported numerical density information. Mitochondria increased in size and elongated from maturation to senescence. The authors suggested that with age, mitochondria undergo morphological adaptations in order to meet metabolic demands. Mitochondrial enlargement with age was also demonstrated in rat myocardium by Cosmas et al. (1994). The authors showed that with maturation there is a shift in mitochondrial size distributions toward a greater percentage of smaller organelles. Senescence was associated with a shift in mitochondrial size distributions toward a greater percentage of enlarged organelles. The shift toward smaller mitochondria during maturation was postulated to increase mitochondrial surface-to-volume ratio, and indicative of enhanced physiological function. During senescence, the shift toward enlarged mitochondria suggests fusion of organelles, and is consistent with a decline in physiological function (Cosmas et al., 1994; Bertoni-Freddari et at., 1993).

Intervention studies have shown that mitochondrial morphology can be altered by exercise and diet. Cosmas and Manfredi (1995) reported that exercise training during maturation and middle age dramatically increase the proportion of smaller size mitochondria in rat myocardium, enhancing tissue oxidative function. Further research by Cosmas and associates (1996) examined the effect of aging and physical training on mitochondrial profiles from the left ventricular apex of male mice. They defined a "threshold age" (450 days) in which initiation of exercise training fails to increase the population of small mitochondria. This is consistent with Kiesling et al. (1973) who examined muscle mitochondrial morphology in young and old physically trained and 
untrained men. They found that both young and old subjects responded to training with an increase in mitochondrial volume. However, the training effect on mitochondrial volume in the young subjects was due to an increase in the number of mitochondria, while the old subjects responded with an increase in mitochondrial size.

Utley and associates (1999) examined the effect of diet restriction initiated at 14 weeks of age on skeletal muscle mitochondria in old (19 months) and older (27 months) mice. They found that forty percent caloric restriction decreased skeletal muscle mitochondrial mean area in older ( 27 months) mice. The caloric restricted mice showed evidence of smaller, more rounded mitochondria when compared to their agematched controls. This supports the hypothesis that caloric restriction maintains mitochondrial plasticity with age.

This present study supports the hypothesis that vitamin E is effective at maintaining mitochondrial plasticity, and this benefit appears to be maintained with age. Liver mitochondrial area was significantly smaller in "old" vitamin E supplemented mice, compared to age-matched controls. Continued vitamin E supplementation maintained mitochondrial size with age, with a slight shift toward larger sizes (Figure 1), associated with elongation (Figure 2).

Epidemiological evidence suggests that vitamin E supplementation may enhance the immune response and reduce the incidence of coronary disease and certain cancers. Vitamin $\mathrm{E}$ is an effective antioxidant, and this present study may be evidence of how vitamin E prevents oxidative damage. Morphometric data from this study implies that vitamin $\mathrm{E}$ prevents the age-related changes in mitochondrial size. It is tempting to 
suggest that vitamin E supplementation protects liver mitochondria from oxidative damage, and enhances cellular respiration. 


\section{Table 1 - Vitamin E and Mitochondrial Morphometry}

$\begin{array}{lllll}\text { CONT } & \text { VIT E } & \text { VIT E } & \text { CvsE } & \text { E OLD vs } \\ \text { OLD } & \text { OLD } & \text { OLDER } & \text { OLD } & \text { E OLDER }\end{array}$

$\bar{N}$

$\begin{array}{llll}\text { Age (days) } & \mathbf{7 6 0} \pm 3.8 & \mathbf{7 5 8} \pm 2.9 & \mathbf{8 2 7} \pm \mathbf{1 7 . 9}\end{array}$

$\begin{array}{llllll}\text { Area }\left(\mu^{2}\right) & .797 \pm .45 & .592 \pm .43 & .582 \pm .25 & \mathbf{P}<.0001 & \text { NS }\end{array}$

Major Axis $(\mu) \quad 1.06 \pm .29 \quad .889 \pm 0.3 \quad .944 \pm .21 \quad$ p $<.0001 \quad$ p $<.002$

$\begin{array}{llllll}\text { Minor Axis }(\mu) & .904 \pm .22 & .766 \pm .26 & .755 \pm .16 & \mathbf{p}<.0001 & \text { NS }\end{array}$ 


\section{Figure 1 - Mitochondrial Area Pooled Cumulative Data}

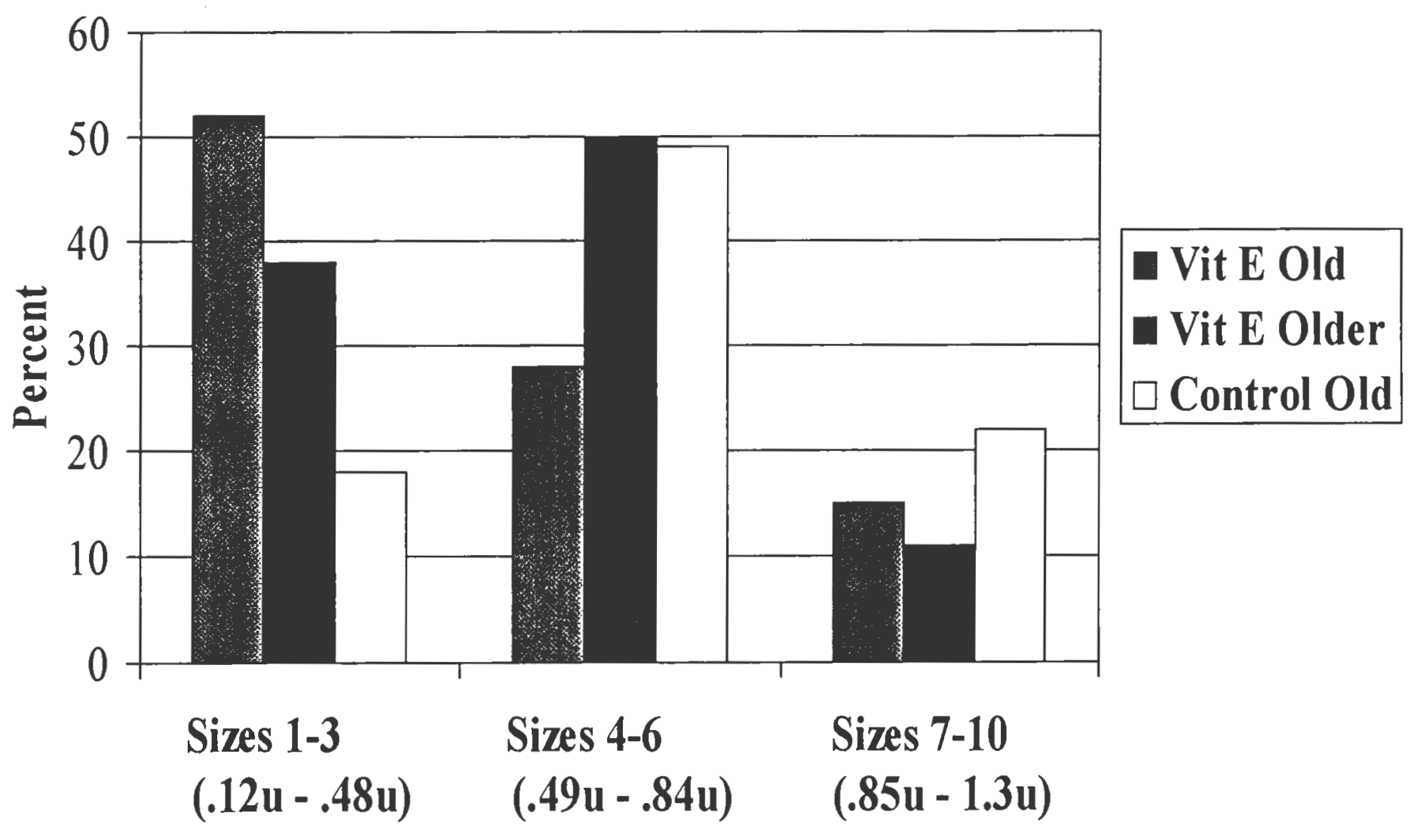




\section{Figure 2 - Mitochondrial Sizes Long Axis - Pooled Data}

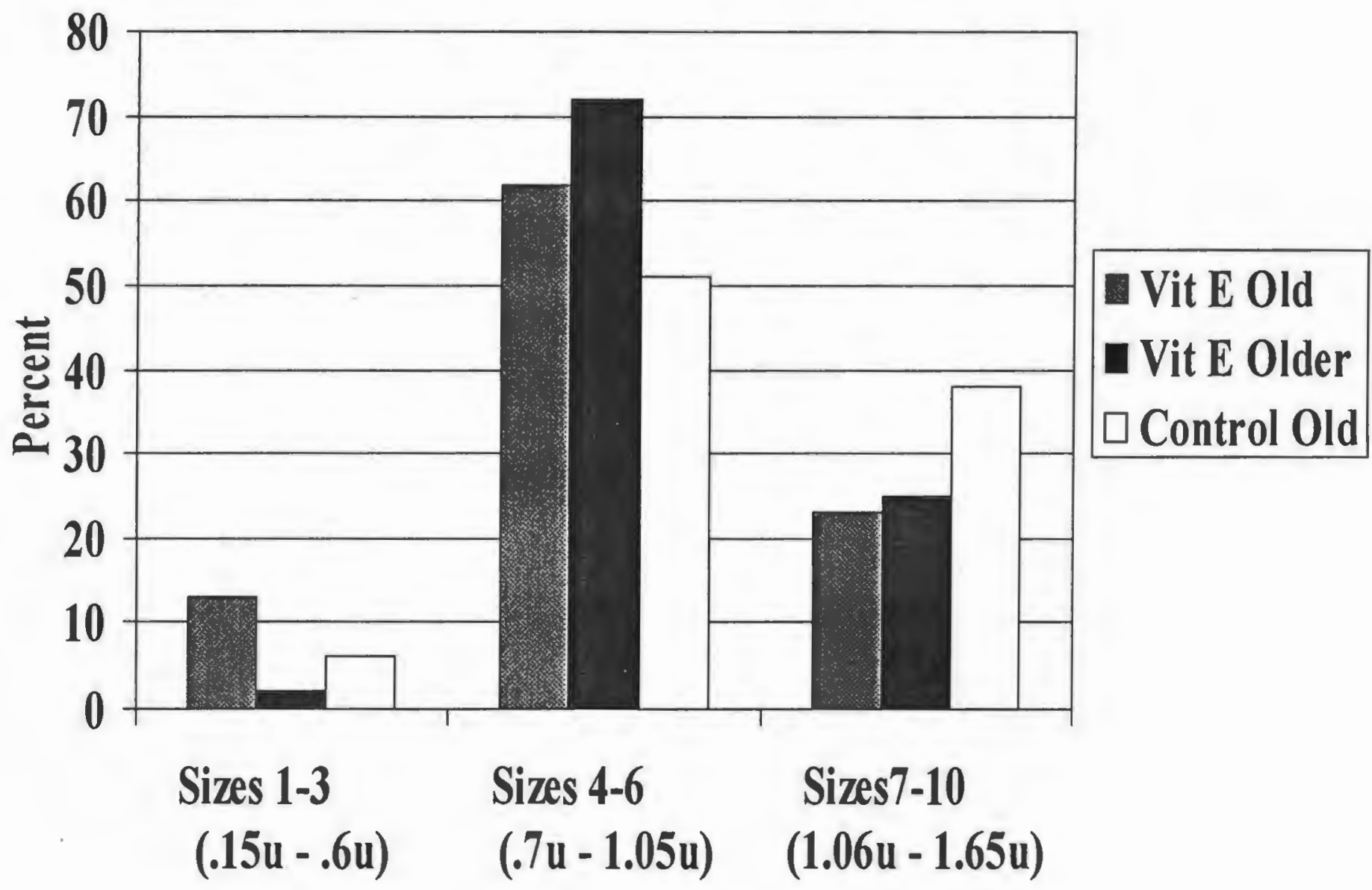




\section{Figure 3 - Mitochondrial Sizes Short Axis - Pooled Data}

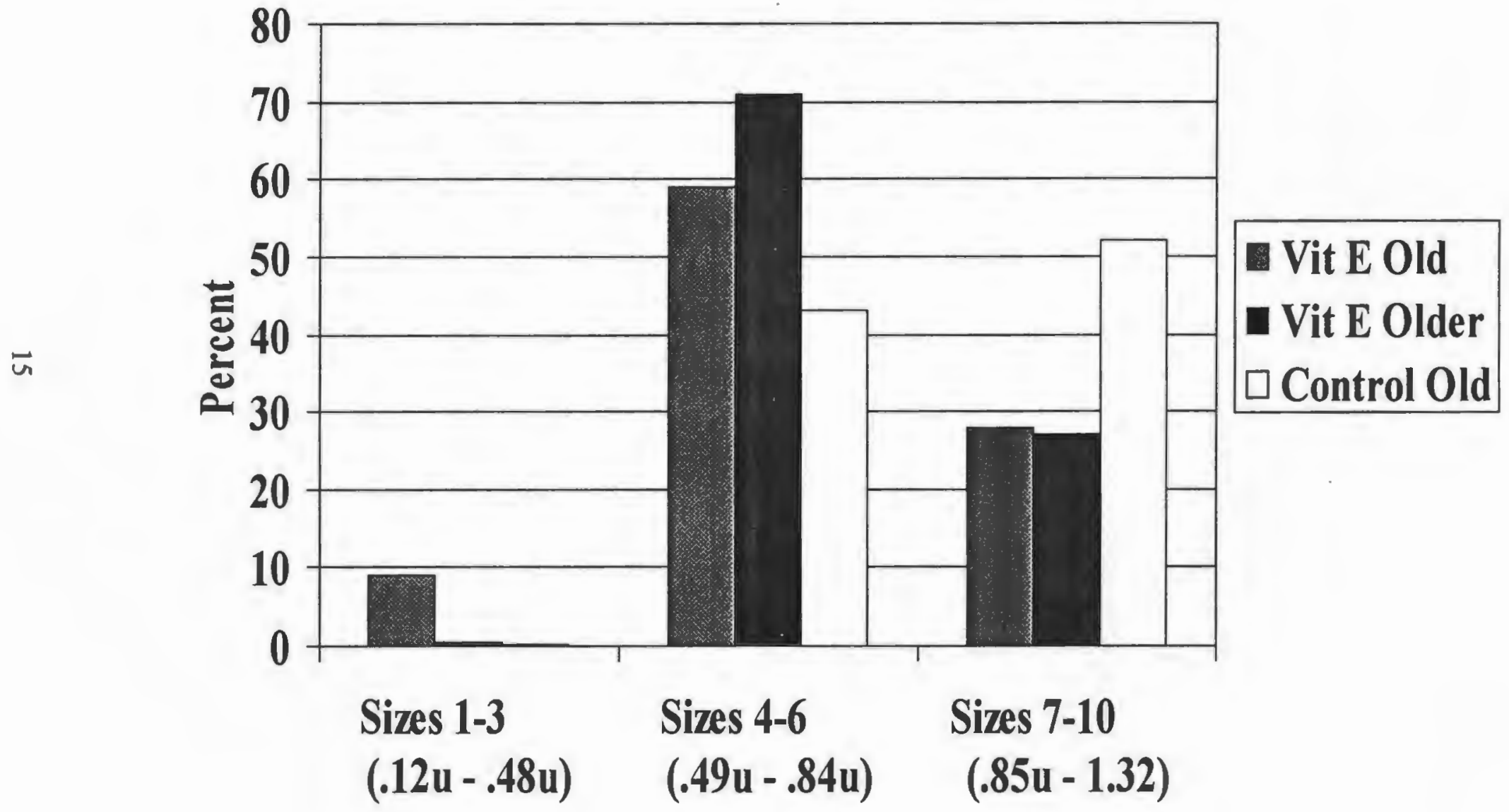




\section{REFERENCES}

Ames, B.N., Shigenaga, M.K., Hagen, T.M. (1993). Oxidants, antioxidants, and the degenerative diseases of aging. Procedures National Academy of Science USA, 90 , 7915-7922.

Baudhuin, P. and Berthet J. (1967). Electron microscopic examination of subcellular fractions. The Journal Of Cell Biology, 35, 631-648.

Bertoni-Freddari, C., Fattoretti, P., Casoli, T., Spagna, C., Meier-Ruge, W., Ulrich, J. (1993). Morphological plasticity of synaptic mitochondria during aging. Brain Research, 628, 193-200.

Cosmas, A.C., Edington, D.W., Manfredi, T.G. (1994). Mitochondrial distributions in hearts of male rats as a function of aging. Age, 17 (4), 158.

Cosmas, A.C., Edington, D.W., McCafferty, W.B., Manfredi, T.G. (1996). Exercise and longevity: Further evidence for a threshold age. Age, 34-35.

Cosmas, A.C., Manfredi, T.G. (1995). The effects of aging and physical exercise on mitochondrial distributions in hearts of male rats. Abstract, American Aging Association, 25th Annual Meeting.

Frigg, M., Rohr, H.P. (1976). Ultrastructural and stereological study on the effect of vitamin $\mathrm{E}$ on liver mitochondrial membranes. Experimental And Molecular Pathology, 24, 236-243.

Ji, L.L., Leichtweis, S. (1997). Exercise and oxidative stress: sources of free radicals and their impact on antioxidant systems. Age, 20, 91-106.

Kiesling, K.H., Pilstrom, L., Karlsson, J., Piehl, K. (1973). Mitochondrial volume in skeletal muscle from young and old physically untrained and trained healthy men and from alcoholics. Clinical Science, 44, 547-554.

Lipman, R.D., Bronson, R.T., Wu, D., Smith, D.E., Prior, R., Cao, G., Han, S.N., Martin, K.R., Meydani, S.N., Meydani, M. (1998). Disease incidence and longevity are unaltered by dietary antioxidant supplementation initiated during middle age in C57BL/6 mice. Mechanisms of Aging and Deveolpment, 103, 269-284.

Meydani, M. (1995). Vitamin E. The Lancet, 345, 170-175. 
Meydani, M., Evans, W.J., Handelman, G., Biddle, L., Fielding, R.A., Meydani, S.N., Burrill, J., Fiatarone, M.A., Blumberg, J.B., Cannon, J.G. (1993). Protective effect of vitamin $\mathrm{E}$ on exercise-induced oxidative damage in young and older adults. American Journal of Physiology, 264, R992-R998.

Meydani, S.N., Hayek, M.G. (1995). Vitamin E and aging immune response. Clinics in Geriatric Medicine, 11(4), 567-575.

Meydani, S.N., Wu, D., Santos, M.S., Hayek, M.G. (1995). Antioxidants and immune response in aged persons: overview of present evidence. American Journal of Clinical Nutrition, 62 (suppl), 1462S-1476S.

Shigenaga, M.K., Hagen, T.M., Ames, B.N. (1994). Oxidative damage and mitochondrial decay in aging. Procedures National Academy of Science USA, 91 (23), 10771-10778.

Utley, S.A., Bairos, L.A., Urso, M.L., Lipman, R., Bronson, R., Cosmas, A., Lamont, L., Manfredi, T. (1998). Abstract presented at the New England American College of Sports Medicine meeting, Providence, RI.

Zorov, D.B. (1996). Mitochondrial damage as a source of diseases and aging: a strategy of how to fight these. Biochimica et Biophysica Acta, 1275, 10-15. 
APPENDIX I 


\section{LITERATURE REVIEW}

\section{MITOCHONDRIA AND AGING}

The rapidly growing elderly population has directed research attention to the mechanisms associated with the aging process. Research has shown that oxidative stress is a major contributor to aging and degenerative diseases (Sohal and Weindruch, 1996). Aging is a consequence of cellular damage to DNA, proteins, and lipids. This damage is caused by oxidants that are by-products of aerobic metabolism and include superoxide, hydrogen peroxide, and hydroxyl radicals.

During cellular respiration, mitochondria consume oxygen and most of the oxygen combines with hydrogen to produce water. A small percentage of consumed oxygen will produce reactive oxygen species as electrons escape from the electron transport chain. These reactive oxygen species have unpaired electrons and are chemically unstable. Reactive oxygen species can cause oxidative damage to the cell as they attempt to become more stable by extracting electrons. (Ames, Shigenaga, and Hagen, 1993; Shigenaga, Hagen, and Ames, 1994). This free radical theory on aging is thought to be primarily due to aging mitochondria (Harmen, 1994).

Mitochondria are the major source of oxidant production because they are the subcellular sites where oxidative phosphorylation takes place. The high oxidative activity of mitochondria make them major targeted sites for oxidative damage (Shigenaga et al., 1994; Zorov, 1996). The cell protects itself by a high rate of mitochondrial turnover, thereby removing damaged mitochondria. Despite the high rate of tissue mitochondrial turnover, mitochondrial function declines with age as a 
result of damage caused by endogenously produced oxidants (Ames et al., 1993; Shigenaga et al., 1994).

Mitochondria undergo morphological changes from maturation to senescence. With age, membranes become rigid making mitochondrial more sensitive to oxidants and susceptible to oxidative damage (Shigenaga et al., 1994). Following maturation, mitochondria show evidence of lipid membrane damage resulting in leakage of cytochrome $\mathrm{C}$ into the cytosol, triggering events leading to apoptosis ( $\mathrm{Ji}$ and Leichtweis, 1997). Other histological changes include a significant decrease in the number of mitochondria, organelle enlargement and elongation (Bertoni-Freddari et al., 1993).

Bertoni-Freddari and associates (1993) examined cerebellar glomeruli of young, adult, and old rats. They investigated mitochondrial volume density, numerical density, average volume, and average length. They found that volume density remained constant throughout life, however numerical density decreased from adult to old rats. Mitochondrial size and shape were significantly altered from maturation to senescence, resulting in greater populations of larger, more elongated mitochondria. These findings suggest that with age, mitochondria undergo morphological adaptations in order to meet metabolic demands. Although mitochondrial enlargement and elongation suggest more mass per unit of tissue area, these mitochondria have lower membrane surface area relative to mitochondrial volume. Larger, more elongated mitochondria are believed to have lower membrane integrity.

Cosmas and associates (1994) examined the mitochondrial size distributions of 
rat myocardium. Their results showed that with maturation there is a shift in mitochondrial size distributions toward a greater percentage of smaller organelles. Senescence was associated with a shift in mitochondrial size distributions toward a greater percentage of enlarged organelles. The shift toward a greater percentage of smaller mitochondria during maturation increases the mitochondrial membrane's surface-to-volume ratio and may indicate enhanced physiological function. During senescence, the shift toward a greater percentage of enlarged mitochondria suggests fusion of organelles and is consistent with a decline in physiological function (Cosmas et al., 1994; Bertoni-Freddari et al., 1993).

As mitochondrial function declines with age, susceptibility to oxidative damage increases. Cellular defense mechanisms protect against oxidative damage by preventing the formation of reactive oxygen species, or by destroying already formed oxidants (Zorov, 1996). These defenses include endogenous enzymatic and dietary antioxidants (Ames et al., 1993). The oxidant-antioxidant balance is important to cell function. When antioxidant defenses are not adequate, cells are under chronic oxidative stress (Meydani et al., 1995).

\section{VITAMIN E SUPPLEMENTATION}

Vitamin $\mathrm{E}$ is an effective antioxidant because it protects against oxidative stress and contributes to cellular membrane stability. Epidemiological evidence suggests that vitamin $\mathrm{E}$ and other antioxidants might decrease the prevalence of cardiovascular disease and certain cancers (Meydani, 1995). Substantial evidence associates the 
oxidation of low-density lipoproteins with the development of atherosclerosis and coronary disease. Vitamin $\mathrm{E}$ is a lipid-soluble antioxidant that increases the resistance of low-density lipoproteins to oxidation and may reduce the incidence of coronary artery disease (Stampfer et al., 1993). Evidence such as this has resulted in research on the relationship between vitamin $\mathrm{E}$ and the incidence of morbidity and mortality.

A study by Losonczy et al. (1996) examined the effect that vitamin E and vitamin $\mathrm{C}$ supplementation had on the risk of all-cause mortality and coronary heart disease mortality in older persons. The subjects of this study were 11,178 individuals aged 67 to 105 years. They participated in the Established Populations for Epidemiologic Studies of the Elderly from 1984 to 1993 . They were followed annually for six years and mortality data was collected for an additional two to three years. The results showed a lower risk of all-cause mortality, coronary heart disease mortality, and cancer mortality in all users of vitamin E supplements compared to nonusers. The reduction in relative risk for cancer mortality was not as significant as the reduction in relative risk for all-cause and coronary disease mortality. These results suggest a protective effect of vitamin $\mathrm{E}$ on all-cause and coronary mortality.

Stampfer et al. (1993) examined the relationship between vitamin E consumption and the risk of coronary disease in women and concluded that women who consumed high levels of vitamin $\mathrm{E}$ had a significantly reduced incidence of major coronary disease than women who consumed low levels of vitamin E. The higher intakes of vitamin $\mathrm{E}$ were achieved through supplements. Women not taking supplements but consuming high levels of vitamin E from their diet alone did not have a 
significant reduction in risk of major coronary disease.

A study by Rimm et al. (1993) was a similar prospective study that looked at the relationship between vitamin $\mathrm{E}$ consumption and the risk of coronary heart disease in men. The results of their data analysis revealed a lower risk of coronary disease in men who had high intakes of vitamin E. The most significant reduction in risk was among men using vitamin $\mathrm{E}$ supplements. However, this study found a moderate reduction in risk of coronary disease in men with higher intakes of vitamin $E$ from dietary sources not using supplements. The benefit of vitamin $\mathrm{E}$ was greater with longer-term use indicating a duration factor. This supports Losonczy et al. (1996) who found the greatest benefit in risk reduction of coronary disease to be in subjects using vitamin E supplements at two points in time.

- These studies suggest a protective effect of vitamin E against the incidence of coronary disease with higher intakes of vitamin $\mathrm{E}$. The greatest benefit is achieved with the use of vitamin E supplements not part of a multivitamin. It is also suggested that the higher levels of vitamin $\mathrm{E}$ achieved through supplement use is necessary to increase the resistance of low-density lipoproteins to oxidation, and reduce the incidence of atherosclerosis and coronary disease. These levels cannot be achieved through dietary vitamin $\mathrm{E}$ only and are well above the recommended dietary allowance.

\section{VITAMIN E AND EXERCISE}

Evidence suggests that strenuous exercise increases production of reactive oxygen species by the mitochondria. This results in an imbalance between reactive 
oxygen and antioxidant defenses which may lead to exercise-induced tissue damage (Ji and Leichtweis, 1997; Dekkers et al., 1996). Further evidence indicates that high intensity resistance training increases free radical production (McBride et al., 1998). A clinical study by Meydani et al. (1993) examined the effect of vitamin E supplementation on exercised induced oxidative damage in humans. Experimental subjects were supplemented with vitamin $\mathrm{E}$ for $\mathbf{4 8}$ days before eccentric exercise to increase vitamin $\mathrm{E}$ in plasma and skeletal muscle. Control subjects received a placebo. Researchers looked at changes in oxidative products and antioxidant status in skeletal muscle, plasma, and urine after eccentric exercise. The authors suggested that vitamin E protects against oxidative damage which is a detrimental effect of exercise. These results were supported by McBride et al. (1998) who examined the effect of vitamin E supplementation on free radical formation and reported vitamin E supplementation may prevent the variables associated with muscle membrane disruption. Exercise is encouraged in all populations for its obvious health benefits. The authors concluded that the negative effects of exercise may be offset by dietary supplementation of antioxidants.

\section{VITAMIN E DEFICIENCY}

Frigg and Rohr (1976) examined the effect of vitamin E deficiency on liver mitochondrial membranes. The subjects of this study were male hybrid mice. These mice received either a vitamin $\mathrm{E}$ deficient diet, a diet supplemented with vitamin $\mathrm{E}$, or a commercial standard diet. The volume density of mitochondria remained constant in all 
groups, but the inner mitochondrial membrane was enhanced with vitamin E deficiency. The surface density of the inner membrane was increased with vitamin E deficiency, but the cytochrome density was reduced. The authors concluded that chronic vitamin E deficiency resulted in an increased formation of defective membranes.

Deficiencies in vitamin E, carotenoids, and glutathione, have been shown to have a harmful effect on the immune response in aged individuals. Antioxidant supplementation to levels above the recommended daily allowance have enhanced immune function in several studies (Meydani and Hayek, 1995). A large scale study conducted by Tufts University examined the effect of long-term dietary supplementation with antioxidants including vitamin $\mathrm{E}$, glutathione, melatonin, and strawberry extract. This study demonstrated that vitamin $\mathrm{E}$ was the only antioxidant effective at reducing the influenza lung viral titer in mice (Meydani et al., 1998). Data from several prospective studies have associated low levels of plasma vitamin E concentration with a high rate of cardiovascular disease. Antioxidant supplementation studies have associated high intakes of vitamin $\mathrm{E}$ with a reduced incidence of coronary events. Epidemiological evidence has suggested that vitamin E may reduce the incidence of certain cancers. Animal studies have indicated that vitamin $\mathrm{E}$ may reduce the incidence of cancer by inhibiting tumor production (Meydani et al., 1995). Dietary supplementation of antioxidants help to counteract the depressed immune response in the aged and may protect against cardiovascular disease and cancer which are the leading causes of death in this country (Meydani, 1995). 


\section{MITOCHONDRIAL INTERVENTION STUDIES}

Intervention studies including diet and exercise can alter mitochondrial morphology. Forty percent caloric restriction has been reported to decrease skeletal muscle mean mitochondrial area in older mice (Utley et al., 1999). The caloric restricted mice showed evidence of smaller, more rounded mitochondria when compared to their age-matched controls. This supports the hypothesis that caloric restriction maintains mitochondrial plasticity with age.

Cosmas and Manfredi (1995) reported that exercise training during maturation and middle age dramatically increased the proportion of smaller size mitochondria in rat myocardium. It is suggested that the decrease in mitochondrial size observed with physical training may represent enhanced mitochondrial function. Further research by Cosmas and associates (1996) examined the effect of aging and physical training on mitochondrial profiles from the left ventricular apex of male mice. They defined a "threshold age" (450 days) in which initiation of exercise training fails to increase the populations of small mitochondria. This is consistent with Kiesling et al. (1973) who examined muscle mitochondrial morphology in young and old physically trained and untrained men. They found that both young and old subjects responded to training with an increase in mitochondrial volume. However, in the young subjects this was due to an increase in the number of mitochondria while the old subjects responded with an increase in mitochondrial size only. These studies suggest that mitochondria lose their ability to divide with age which results in a greater percentage of enlarged mitochondria. 


\section{SUMMARY}

Ageing and degenerative disease is a consequence of cellular damage to DNA, proteins, and lipids. The cellular damage is caused by reactive oxygen species which are by-products of normal cellular respiration. Mitochondria are the primary sites of oxidative phosphorylation and are the major source of reactive oxygen species. They are also major targeted sites for oxidative damage. Oxidative damage to mitochondria results in a decline in mitochondrial function and increased oxidative stress to cellular components. When antioxidant mechanisms are not adequate to protect the cell against oxidative stress there is increased formation of reactive oxygen species and cellular damage occurs. This free radical theory on aging has resulted in many investigations involving mitochondria.

Research on mitochondrial morphology has shown that mitochondria undergo age-related changes in size and shape. Changes include enlargement and elongation of these organelles. Researchers have suggested that these changes in mitochondrial morphology are due to an inability of mitochondria to divide with age resulting in fewer, enlarged mitochondria. This shift toward a greater percentage of enlarged organelles is consistent with a decline in mitochondrial function and an increased susceptibility to oxidative damage. Intervention studies have shown that mitochondrial morphology can be effected by interventions such as diet and exercise. Caloric restriction has been shown to prevent the age-related changes that occur in mitochondria and may be beneficial in maintaining mitochondrial plasticity with age. Deficiencies in vitamin $E$ have resulted in increased formation of defective 
mitochondrial membranes. Physical training up to a certain age results in smaller, more rounded mitochondria. Preventing the age-related changes in mitochondria may result in increased efficiency of cellular respiration and protection against cellular oxidative damage. 
APPENDIX II 


\section{STATEMENT OF THE PROBLEM}

The purpose of this study was to examine mitochondrial profiles from the liver of mice in response to vitamin E supplementation. This study analyzed mitochondrial size distributions in the liver of "old" (760 days) controls and vitamin E supplemented mice and "older" (827 days) vitamin E supplemented mice.

\section{Hypotheses}

The following hypotheses regarding the effect of vitamin E supplementation and age on mitochondrial morphometry were tested in this study:

1. The mean mitochondrial size of the "old" mice fed only modified AIN76 will be greater than that of the age-matched mice supplemented with vitamin $\mathrm{E}$.

2 "Old" mice fed only modified AIN76 will have a greater percentage of larger mitochondria when compared to the age-matched mice supplemented with vitamin $\mathrm{E}$.

3 "Old" mice fed only modified AIN76 will have a greater percentage of elongated mitochondria when compared to the age-matched mice supplemented with vitamin $\mathrm{E}$.

4 Age will have no effect on mitochondrial size in mice fed AIN76 diet supplemented with vitamin $\mathrm{E}$.

5 Age will have no effect on mitochondrial shape in mice fed AIN76 diet supplemented with vitamin $\mathrm{E}$. 


\section{Evaluation of Hypotheses}

1. The mean mitochondrial size of the "old" mice fed only modified AIN76 will be greater than that of the age-matched mice supplemented with vitamin $\mathrm{E}$.

Mean mitochondrial area was evaluated for "old" control and "old" vitamin E supplemented mice. An Analysis of Variance model for pooled data was used to test for significant differences due to diet. The "old" vitamin E supplemented mice had significantly smaller $(\mathrm{p}<.0001)$ liver mitochondria than age-matched controls.

Therefore, this hypothesis was accepted.

2. "Old" mice fed only modified AIN76 will have a greater percentage of larger mitochondria when compared to the age-matched mice supplemented with vitamin $\mathrm{E}$.

Size distributions for mitochondrial area revealed that mice fed only AIN76 had a greater percentage of "large" mitochondria when compared to the "old" vitamin E supplemented mice. The "old" vitamin E supplemented mice had 15\% of their population fall into this category compared to $22 \%$ for age-matched controls. The "old" vitamin E supplemented mice had $52 \%$ of their total mitochondrial population fall into the "small" category compared to $18 \%$ for age-matched controls. This study showed that vitamin $\mathrm{E}$ supplementation resulted in a shift in size distribution from "medium" and "large" size mitochondria to "small" and "medium" size mitochondria. Therefore, this hypothesis was accepted.

3. "Old" mice fed only modified AIN76 will have a greater percentage of elongated mitochondria when compared to the age-matched mice supplemented with vitamin $\mathrm{E}$. 
Size distributions for long axis showed that mice fed only AIN76 had $38 \%$ of their population fall into the "large" category compared to $24 \%$ for "old" vitamin E supplemented mice. An Analysis of Variance model for pooled data revealed that the "old" controls had a significantly larger $(\mathrm{p}<.0001)$ long axis than age-matched mice supplemented with vitamin E. However, a similar trend was found for the short axis. The ratios for long axis to short axis were calculated for each mitochondria evaluated. A t-test was performed to test for significance, and revealed no significant effect of vitamin E. Therefore, this hypothesis was rejected.

4. Age will have no effect on mitochondrial size in mice fed AIN76 diet supplemented with vitamin $\mathrm{E}$.

Mean mitochondrial area was evaluated for "old" and "older" vitamin $\mathrm{E}$ supplemented mice. An Analysis of Variance model for pooled data was used to test for significant differences due to age. Age had no effect on the mean mitochondrial area in mice fed AIN76 diet supplemented with vitamin E. Therefore, this hypothesis was accepted.

5. Age will have no effect on mitochondrial shape in mice fed AIN76 diet supplemented with vitamin $\mathrm{E}$.

Age had a significant effect $(\mathrm{p}<.002)$ on the long axis in the vitamin $\mathrm{E}$ supplemented mice, but no significant effect on the short axis. Analysis of the ratios for the long axis to short axis using a t-test showed that age had a significant effect on mitochondrial elongation in vitamin E supplemented mice. Therefore, this hypothesis was rejected. 


\section{Delimitations}

The scope of this study included the following delimitations:

1. Twelve C57BL/6 mice were the animals used in this study.

2. The mice were 760 days ("old") and 827 days ("older") of age.

3. A control group was used.

4. Experimental and control animals were male.

5. Liver mitochondria was analyzed.

6. Measurements included mitochondrial area, long axis, and short axis.

\section{Limitations}

The limitations of this study were:

1. Sample size was small and may not be representative of the total population.

2. Whole liver tissue was not used.

3. The "older" vitamin E supplemented mice did not have an age-matched control group. 


\section{BIBLIOGRAPHY}

Ames, B.N., Shigenaga, M.K., Hagen, T.M. (1993). Oxidants, antioxidants, and the degenerative diseases of aging. Procedures National Academy of Science USA, 90, 7915-7922.

Bertoni-Freddari, C., Fattoretti, P., Casoli, T., Spagna, C., Meier-Ruge, W., Ulrich, J. (1993). Morphological plasticity of synaptic mitochondria during aging. Brain Research, 628, 193-200.

Cosmas, A.C., Edington, D.W., Manfredi, T.G. (1994). Mitochondrial distributions in hearts of male rats as a function of aging. Age, 17 (4), 158.

Cosmas, A.C., Edington, D.W., McCafferty, W.B., Manfredi, T.G. (1996). Exercise and longevity: Further evidence for a threshold age. Age, 34-35.

Cosmas, A.C., Manfredi, T.G. (1995). The effects of aging and physical exercise on mitochondrial distributions in hearts of male rats. Abstract, American Aging Association, 25th Annual Meeting.

Dekkers, J.C., van Doornen, L.J., Kemper, H.C. (1996). The role of antioxidant vitamins and enzymes in the prevention of exercise-induced muscle damage. $\underline{\text { Sports }}$ Medicine, 21, 213-238.

Frigg, M., Rohr, H.P. (1976). Ultrastructural and stereological study on the effect of vitamin $\mathrm{E}$ on liver mitochondrial membranes. Experimental And Molecular Pathology, 24, 236-243.

Harman, D. (1994). Aging: Prospects for further increases in the functional life span. Age, 17, 119-146.

Ji, L.L., Leichtweis, S. (1997). Exercise and oxidative stress: sources of free radicals and their impact on antioxidant systems. Age, 20, 91-106.

Kiesling, K.H., Pilstrom, L., Karlsson, J., Piehl, K. (1973). Mitochondrial volume in skeletal muscle from young and old physically untrained and trained healthy men and from alcoholics. Clinical Science, 44, 547-554.

Losonczy, K.G., Harris, T.B., Havlik, R.J. (1996). Vitamin E and vitamin C supplement use and risk of all-cause and coronary heart disease mortality in older persons: the established populations for epidemiologic studies of the elderly. American Journal of Clinical Nutrition, 64, 190-196. 
McBride, J.M., Kraemer, W.J., Triplett-McBride T, Sebastianelli W. (1998). Effect of resistance exercise on free radical production. Medical Science Sports Exercise, 30, 67-72.

Meydani, M. (1995). Vitamin E. The Lancet, 345, 170-175.

Meydani, M., Evans, W.J., Handelman, G., Biddle, L., Fielding, R.A., Meydani, S.N., Burrill, J., Fiatarone, M.A., Blumberg, J.B., Cannon, J.G. (1993). Protective effect of vitamin $E$ on exercise-induced oxidative damage in young and older adults. American Journal of Physiology, 264, R992-R998.

Meydani, M., Lipman, R.D., Han, S.N., Wu, D., Beharka, A., Martin, K.R., Bronson, R., Cao, G., Smith, D., Meydani, S.N. (1998). The effect of long-term dietary supplementation with antioxidants. Annals of the New York Academy of Sciences, $\underline{854}, 352-360$.

Meydani, S.N., Hayek, M.G. (1995). Vitamin E and aging immune response. Clinics in Geriatric Medicine, 11(4), 567-575.

Meydani, S.N., Wu, D., Santos, M.S., Hayek, M.G. (1995). Antioxidants and immune response in aged persons: overview of present evidence. American Journal of Clinical Nutrition, 62 (suppl), 1462S-1476S.

Rimm, E.B., Stampfer, M.J., Ascherio, A., Giovannucci, E., Colditz, G.A., Willet, W.C. (1993). Vitamin E consumption and the risk of coronary heart disease in men. The New England Journal Of Medicine, 328, 1450-1456.

Shigenaga, M.K., Hagen, T.M., Ames, B.N. (1994). Oxidative damage and mitochondrial decay in aging. Procedures National Academy of Science USA, 91 (23), 10771-10778.

Sohal, R.S., Weindruch, R. (1996). Oxidative stress, caloric restriction, and aging. Science, 273 (5271), 59-63.

Stampfer, M.J., Hennekens, C.H., Manson, J.E., Colditz, G.A., Rosner, B., Willett, W.C. (1993). Vitamin E consumption and the risk of coronary disease in women. The New England Journal Of Medicine, 328, 1444-1449.

Utley, S., (1997) URI Master of Science Thesis.

Zorov, D.B. (1996). Mitochondrial damage as a source of diseases and aging: a strategy of how to fight these. Biochimica et Biophysica Acta, 1275, 10-15. 